\title{
Tissue characteristics and evolution after steam pop
}

\author{
Gaetano Fassini ${ }^{1} \cdot$ Sergio Conti ${ }^{1} \cdot$ Gianluca Pontone $^{2} \cdot$ Mauro Pepi $^{2} \cdot$ Claudio Tondo $^{1}$. \\ Antonio Dello Russo ${ }^{1}$
}

Received: 20 February 2015 / Accepted: 13 March 2015 /Published online: 30 April 2015

(C) Springer Science+Business Media New York 2015

Steam pop can lead to deep tissue disruption and potential cardiac perforation and tamponade. Intracardiac echocardiography (ICE) improves the safety of complex procedures. This is the first report in which ICE allows direct visualization of intramyocardial steam formation and the evolution over the time. Although visualization of steam pop has been described $[1,2]$, no direct real-time tissue change evolution was reported. A patient with monomorphic, right bundle and leftward axis, sustained ventricular tachycardia underwent catheter ablation. During radiofrequency delivery, immediately after a low-tone pop and scattered microbubble visualization, simultaneously with impedance rise, ICE showed a growing, hyperechogenic intramyocardial bubble at the catheter-tissue interface. Radiofrequency was suddenly stopped. The volume of this formation slowly decreased and completely disappeared (Fig. 1, Movie 1-3). A cardiac magnetic resonance showed, at the site of ablation, the presence of edema (T2-weighted). Post-contrast late gadolinium enhancement images showed a bright signal in the same region of edema suggestive for epicardial fibrosis and subendocardial focal rounded hyperenhancement surrounded by a dark rim suggestive for endocardial lesion (Fig. 2).

Electronic supplementary material The online version of this article (doi:10.1007/s10840-015-9997-0) contains supplementary material, which is available to authorized users.

\section{Gaetano Fassini}

gfassini@ccfm.it

1 Cardiac Arrhythmia Research Centre, Centro Cardiologico Monzino IRCCS, via Carlo Parea 4, 20138 Milan, Italy

2 Centro Cardiologico Monzino IRCCS, Milan, Italy

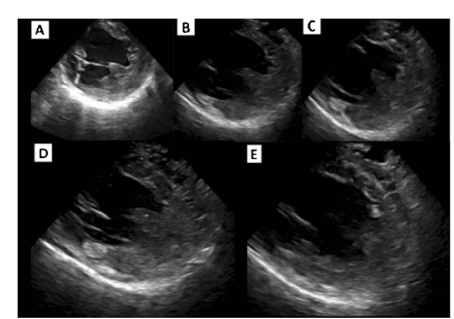

Fig. 1 Intracardiac echocardiography: the probe is positioned in the RV through the tricuspid valve with proper rotation of the transducer; a truncated long-axis view of the LV is obtained. Ablation catheter at the target area is shown in a. During RF, a growing, hyperechogenic intramyocardial formation appears at ICE imaging (b-d). After interruption of RF, the finding disappeared within $1 \mathrm{~min}(\mathbf{e})$

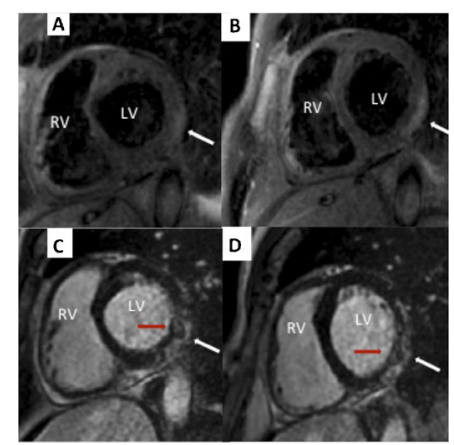

Fig. 2 Cardiac magnetic resonance: triple inversion recovery T2weighted images of basal (a) and middle (b) short axis view of LV showing an epicardial bright signal (white arrow) suggestive for edema with a non-ischemic pattern. Post-contrast late gadolinium enhancement images of basal (c) and middle (d) short-axis view of LV showing bright signal (white arrow) in the same region of edema suggestive for epicardial fibrosis and subendocardial focal rounded hyperenhancement surrounded by a dark rim (red arrow) suggestive for endocardial lesion

\section{References}

1. Tokuda, M., Tedrow, U. B., \& Stevenson, W. G. (2013). Silent steam pop detected by intracardiac echocardiography. Heart Rhythm, 10, 1558-1559.

2. Cochet, H., Sacher, F., Chaumeil, A., \& Jais, P. (2014). Steam pop during radiofrequency catheter ablation: imaging features on magnetic resonance imaging and multidetector computed tomography. Circ Arrhythm Electrophysiol, 7(3), 559-560. 